\title{
AL amyloidosis associated with multiple myeloma revealed by peripheral bilateral polyarthritis: a case report
}

Rova Malala Fandresena Randrianarisoa ${ }^{1}$, Efrasie Rafanomezantsoa ${ }^{1}$, Hitsy Andraina Razafindrazaka $^{1}$, Mihaja Raberahona ${ }^{1}$, Volatiana Andriananja ${ }^{1}$, Stéphanie Norotiana Andriamiharisoa $^{2}$, Fidiarivony Ralison ${ }^{2}$, Hanta Marie Danielle Vololontiana ${ }^{1}$, and MAMY RANDRIA $^{1}$

${ }^{1}$ University of Antananarivo Faculty of Medicine

${ }^{2}$ University of Mahajanga Faculty of Medicine

January 11, 2021

\begin{abstract}
Amyloid arthropathy is often underdiagnosed. The articular forms of AL amyloidosis may mimick the rheumatoid arthritis. The presence of macroglossia should draw attention to chronic polyathralgia.
\end{abstract}

\section{AL amyloidosis associated with multiple myeloma revealed by peripheral bilateral polyarthri- tis: a case report}

Rova Malala Fandresena Randrianarisoa ${ }^{1}$ | Efrasie Rafanomezantsoa ${ }^{2}$ | Hitsy Andraina Razafindrazaka ${ }^{1}$ | Mihaja Raberahona ${ }^{2} \mid$ Volatiana Andriananja $^{2} \mid$ Stéphanie Norotiana Andriamiharisoa $^{3}$ | Fidiarivony Ralison ${ }^{3}$ | Hanta Marie Danielle Vololontiana ${ }^{1}$ | Mamy Jean de Dieu Randria ${ }^{2}$

${ }^{1}$ Department of Internal Medicine, University Hospital of Joseph Raseta Befelatanana, Antananarivo, Madagascar

${ }^{2}$ Department of Infectious Diseases, University Hospital of Joseph Raseta Befelatanana, Antananarivo, Madagascar

${ }^{3}$ Department of Internal Medicine, University Hospital of Mahavoky Atsimo, Mahajanga, Madagascar

Correspondence

Rova Malala Fandresena Randrianarisoa,

Department of Internal Medicine (PSB),

University Hospital of Joseph Raseta Befelatanana

Antananarivo, Madagascar

Email: rrmf7763@gmail.com

\section{ABSTRACT}

Amyloid arthropathy is often underdiagnosed. The articular forms of AL amyloidosis may mimick the rheumatoid arthritis. The presence of macroglossia should draw attention to chronic polyathralgia.

\section{KEYWORDS}


AL amyloidosis, macroglossia, multiple myeloma, rheumatoid arthritis

\section{KEY CLINICAL MESSAGE}

AL amyloidosis can cause peripheral polyarthritis. The presence of macroglossia and absence of antibodies against rheumatoid arthritis may be suggestive of amyloidosis. The search for macroglossia is essential for all chronic polyarthralgia.

\section{INTRODUCTION}

AL amyloidosis is related to extracellular deposition of free light chains (more rarely heavy chains) of monoclonal immunoglobulins produced by a lymphoplasmocytic population. It is secondary to multiple myeloma (MM) in 5 to $15 \%$ of cases and often underdiagnosed due to its polymorphic presentation [1]. The arthropathy secondary to amyloid depositions remains rare with a prevalence of $3 \%$ according to studies [2]. We report a case of AL amyloidosis complicating MM revealed by bilateral peripheral polyarthritis associated with macroglossia.

\section{OBSERVATION}

A 50-year-old male had come for consultation for hands and wrists bilateral joint pain that had been evolving since 2 years, affecting the metacarpophalangeal (MCP) and proximal interphalangeal (PIP) joints, sometimes waking him up at night, not calmed at rest, with gradual increase in intensity. The whole thing was happening in a context of asthenia, anorexia and weight loss. In his history, he reported repeated episodes of infectious lung disease.

The clinical examination at admission revealed a reducible deformation of the hands such as cubital gale of the fingers, flexion of the MCP and PIP (Figure 1A ). There was no joint swelling or skin nodule in relation to the joints. The examination also found a painless macroglossia with ulcerations and indentations of the lateral edges (Figure 1B ) hindering swallowing during feeding.

The biological balance showed an increase in the sedimentation rate to , a C-reactive protein at $59 \mathrm{mg} / \mathrm{l}$, a normocytic anemia at $9 \mathrm{~g} / \mathrm{dl}$ of hemoglobin. The corrected serum calcium was very high at $3.1 \mathrm{mmol} / \mathrm{l}$ with normoprotidemia $(69 \mathrm{~g} / \mathrm{l})$ and serum creatinine was $82 \mu \mathrm{mol} / \mathrm{l}$. With regard to immunological examinations, serum protein electrophoresis showed hypogammaglobulinemia at $6.35 \mathrm{~g} / \mathrm{l}$ with the presence of a kappa light chain monoclonal band on the immunofixation of serum and urinary proteins (Figure 2 ). Total proteinuria was $7 \mathrm{~g} / 24 \mathrm{~h}$ with high Bence Jones proteinuria. Furthermore, the tests for anti-nuclear, anti-native DNA, anti-ENA, anti-fillagrine and anti-citrullin antibodies were negative. Cytological examination from sternal puncture highlighted dystrophic plasma cells infiltration at $10 \%$.

X-rays of the hands (Figure 3 ) and pelvis (Figure 4 ) showed diffuse bone demineralization without erosion or involvement of joint spaces.

The diagnosis of light chains MM was retained in front of medullar plasmocytosis, hypercalcemia, anemia, bone demineralization, absence of monoclonal peak and presence of kappa light chains at immunoelectrophoresis. Assuming an associated amyloidosis, a biopsy of the minor salivary glands was carried out and the histological examination found amorphous eosinophilic material, colored by red Congo (Figure 5 ) and taking on a birefringent aspect to polarized light. It was therefore an AL amyloidosis complicating a kappa light chain MM presenting with rheumatoid pseudoarthritis and macroglossia.

Multidisciplinary care was planned. Our patient had given up the treatment and 3 months later, he had deceased. The cause of death has not been determined.

\section{DISCUSSION}

The most common form of amyloidosis is AL form. The diagnosis is essentially anatomopathological by the identification of extracellular depositions colored in red Congo with dichroism and birefringence yellowgreen characteristic in polarized light. In our case, amyloidosis was confirmed by biopsy of the minor salivary glands. MM was diagnosed by the presence of medullar plasmocytosis associated with anemia, hypercalcemia 
and bone involvement according to the criteria of the International Myeloma Working Group revised in 2014 [3].

AL amyloidosis may mimic several systemic pathologies. Skin depositions may have a sclerosis-like appearance. The damage to the salivary glands can manifest itself as a xerostomia evoking Sjögren's syndrome. We may also encounter joint deformations similar to rheumatoid arthritis (RA) as found in our case, secondary to synovial and periarticular infiltration [4].

Amyloid joint involvement during MM remains rare with a prevalence of $3 \%$ in the Frautel et al series and 2 to $5 \%$ according to Gertz and Kyle [2,5]. Generally, AL amyloidosis arthropathy appears during the evolution of a known myeloma and exceptionally revealing, as in our case. The frequently encountered picture is that of a symmetrical bilateral polyarthritis with progressive installation and rheumatoid appearance. The flexor tendons damage of the fingers can cause a disabling bending attitude. An aspect of swelling or nodule secondary to amyloid infiltration of the soft tissues in regard to the joints can be found [6,7]. Axial involvement is possible, such as atlantoaxial luxation reported by Frautel et al [2]. The quasi-pathognomonic articular sign of amyloidosis is the epaulette aspect of the scapular belt, called shoulder pad sign [8]. Radiographic signs of amyloid arthropathies are often absent or non-specific. When they exist, they consist of either bone erosions, or juxta-articular geodes, or bone demineralization or thickening of the soft tissues.

The articular forms of AL amyloidosis are very varied and may suggest a possible RA. Some semiological elements should not be overlooked, such as the presence of macroglossia and the absence of RA antibodies. The peculiarity of this observation is the clinical predominance of chronic polyarthralgia. According to the literature, several cases of amyloid arthropathy have been mistakenly diagnosed with RA [9]. Clinical assessment takes precedence over classification criteria. The diagnosis of seronegative RA associated with AL amyloidosis had not been discussed as this association has only been described during AA amyloidosis [10].

\section{CONCLUSION}

The clinical presentation of this patient is similar to RA with the exception of the autoantibodies negativity and shows the importance of AL amyloidosis in differential diagnosis of chronic polyarthralgia. The search for macroglossia is essential in the face of any suspicion of systemic pathology and biopsy plays an important role in supporting the diagnosis. This observation illustrates the importance of serum protein electrophoresis and calcemia in the face of any chronic polyarthralgia.

\section{AKNOWLEDGEMENTS}

The authors thank the Pasteur Institute of Madagascar for sending the biopsy piece to the Center of Pathological Morphology of Brussels for histological reading.

\section{CONFLICT OF INTEREST}

The authors declare that there is no conflict of interest regarding the publication of this article.

\section{AUTHOR CONTRIBUTIONS}

RMF Randrianarisoa, E Rafanomezantsoa and HA Razafindrazaka: followed up the patient, collected the clinical data and drafted the report. M Raberahona, V Andriananja and SN Andriamiharisoa: designed and critically revised the report. F Ralison, HMD Vololontiana and MJD Randria: validated the report. All the authors have read and approved the final draft of the manuscript.

\section{ETHICAL APPROVAL}

This article does not contain any personal information that could identify the patient. The names and dates on the X-ray films have been hidden. The authors have included only information necessary for scientific understanding.

\section{REFERENCES}


1. Raubenheimer EJ, Dauth J, Pretorius FJ. Multiple myeloma and amyloidosis of the tongue. J Oral Pathol Med . 1988; 17(9-10): 554- 559.

2. Fautrel B, Fermand JP, Sibilia J, Nochy D, Rousselin B, Ravaud P. Amyloid arthropathy in the course of multiple myeloma. J Rheumatol. 2002; 29(7): 1473- 1481.

3. Rajkumar SV, Dimopoulos MA, Palumbo A, et al. International myeloma working group updated criteria for the diagnosis of multiple myeloma. The Lancet Oncol . 2014; 15(12): e538- 548.

4. Fujishima M, Komatsuda A, Imai H, Wakui H, Watanabe W, Sawada K. Amyloid arthropathy resembling seronegative rheumatoid arthritis in a patient with IgD-kappa multiple myeloma. Intern Med . 2003; 42(1): 121- 124.

5. Gertz MA, Kyle RA. Primary systemic amyloidosis- a diagnostic primer.Mayo Clin Proc . 1989; 64(12): 1505- 1519.

6. Falk RH, Comenzo RL, Skinner M. The systemic amyloidoses. N Engl J Med. 1997; 337(13): 898- 909.

7. Lafforgue P, Senbel E, Figarella-Branger D, et al. Systemic amyloidosis AL with temporal artery involvement revealing lymphoplasmatic malignancy in a man presenting as polymyalgia rheumatica. Ann Rheum Dis. 1993; 52(2): 158- 160.

8. Katz GA, Peter JB, Pearson CM, Adams WS. The shoulder pad sign- a diagnostic feature of amyloid arthropathy. N Engl J Med. 1973; 288(7): 354- 355.

9. Elsaman AM, Radwan AR, Akmatov MK, et al. Amyloid arthropathy associated with multiple myeloma: a systematic analysis of 101 reported cases. Semin Arthritis Rheum. 2013; 43(3): 405- 412.

10. Hazenberg BP, van Rijswijk MH. Aspects cliniques de l'amylose AA. In: Grateau G, Benson MD, Delpech M. Les amyloses. Paris: Flammarion Médecine-Sciences; 2000; 377- 427.

\section{FIGURE LEGEND}

\section{Figure 1}

Photographs of the patient showing characteristic deformation of the hand (A ) and macroglossia (B )

\section{Figure 2}

Serum protein electrophoresis showing absence of monoclonal peak with hypogammaglobulinemia (A ). Presence of monoclonal band on the kappa light chain track to the immunofixation of serum (B ) and urinary $(\mathbf{C})$ proteins on agarose ge

\section{Figure 3}

X-ray of the right (D) and left (G ) hand showing diffuse bone demineralization

\section{Figure 4}

$\mathrm{X}$-ray of the pelvis showing diffuse bone demineralization

\section{Figure 5}

Histological section (Congo red stain and zoom): positivity of amyloid deposits to Congo red 

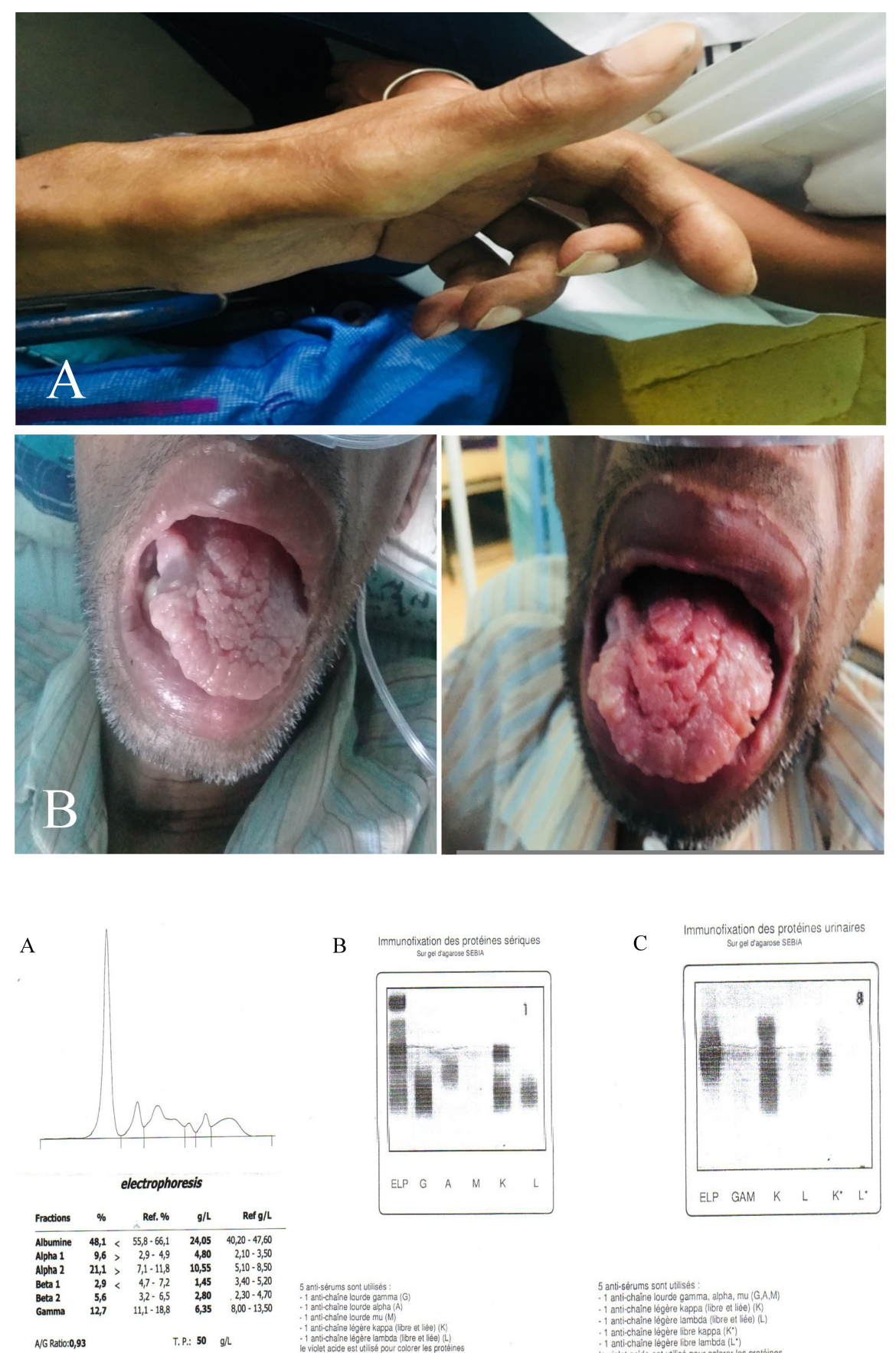

A/G Rati:0,93

T.P.: 50 g/h

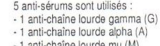

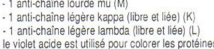

5 anti-sérums sont utilisès:
-1 anti-chaine lourde gamma, apha, mu (G,A,M)

antichaine légère kappa (libre et liee) (K)

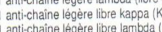



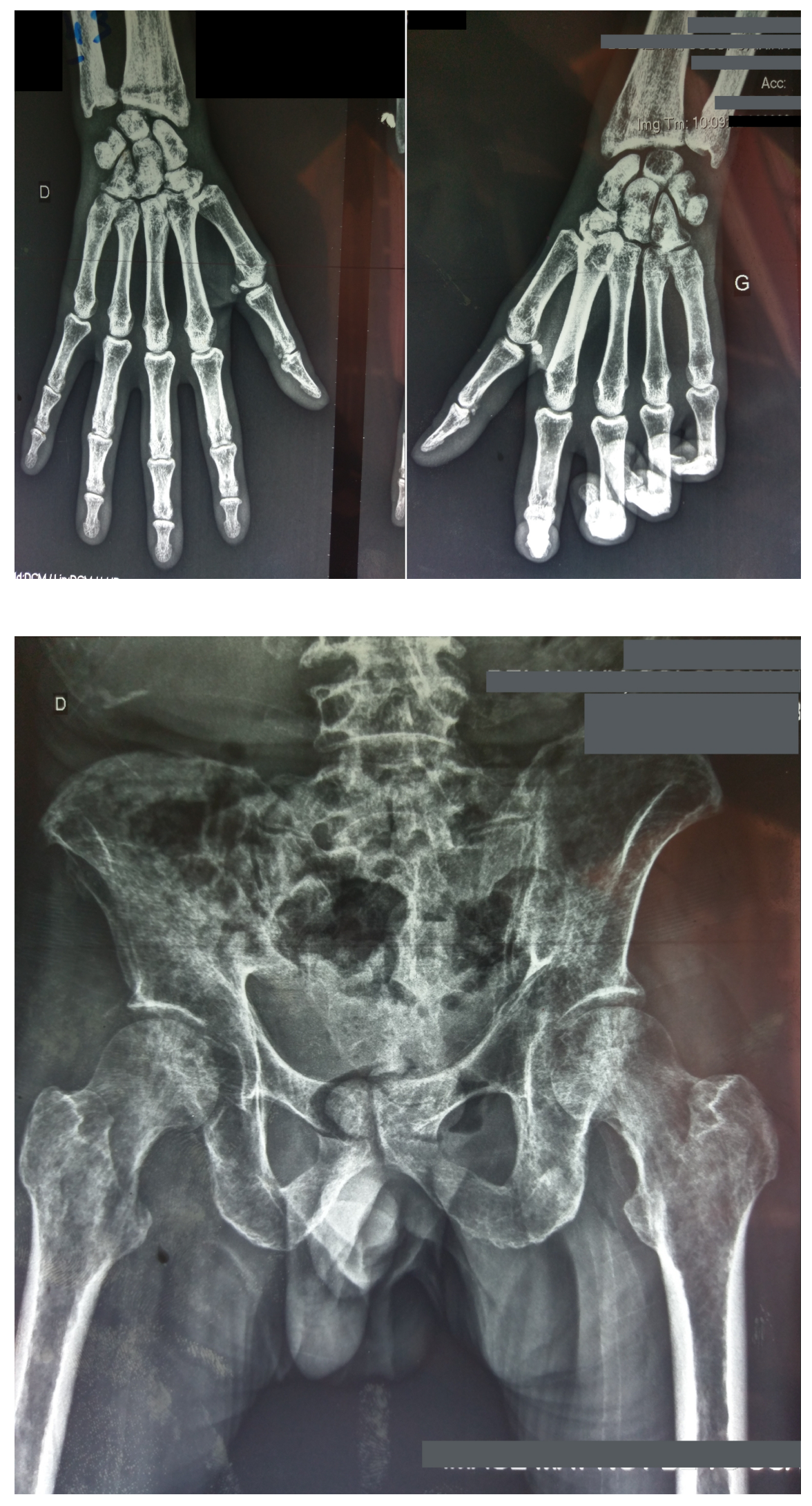


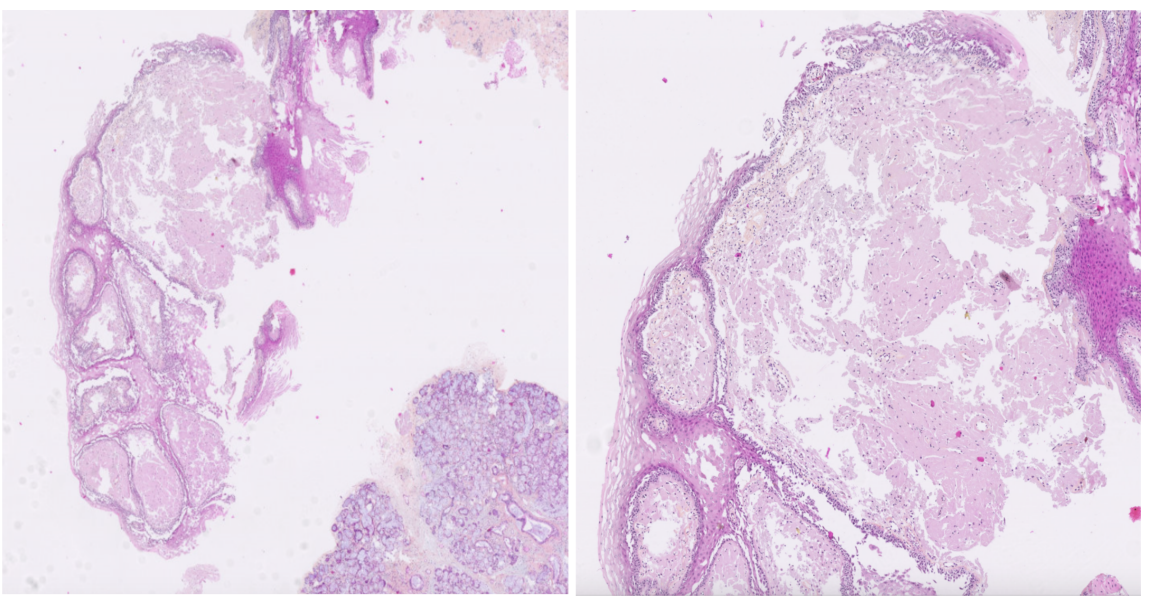

